CASE STUDY

OPIS PRZYPADKU

\title{
AN ANALYSIS OF EARLY MORNING ACTH LEVELS IN THE FIRST CASE OF PEMBROLIZUMAB-INDUCED ADRENALITIS AS A DELAYED IMMUNE-RELATED EVENT (DIRE) - CASE STUDY
}

DOI: $10.36740 /$ WLek202002136

\author{
Igor Alexander Harsch ${ }^{1}$, Anastasiia Gritsaenko ${ }^{1}$, Peter Christopher Konturek ${ }^{2}$ \\ 'DEPARTMENT OF INTERNAL MEDICINE II, DIVISION OF ENDOCRINOLOGY AND METABOLISM, THURINGIA CLINII"GEORGIUS AGRICOLA", SAALFELD/SAALE, GERMANY \\ 2DEPARTMENT OF INTERNAL MEDICINE II, DIVISION OF GASTROENTEROLOGY, THURINGIA CLINIC "GEORGIUS AGRICOLA", SAALFELD/SAALE, GERMANY
}

\begin{abstract}
The aim: The levels of adrenocorticotrophic hormone (ACTH) are elevated in primary adrenal failure (Addison's disease) with a peak in the early morning hours. This also occurs under hydrocortisone replacement therapy due to the unphysiological substitution regime. The aim was to study ACTH levels under two different replacement regimens. This is exemplified in a patient with adrenalitis after immunotherapy for malignant melanoma (MM), since (elevated) levels of ACTH and its cleavage product alpha-melanocyte stimulating hormone (a-MSH) raise concerns since receptors for both hormones can be expressed in melanoma cells.

Material and methods: A female with MM had immunotherapy with pembrolizumab and developed adrenalitis with Addison crisis about one year after discontinuation of this therapy (delayed immune-related event = DIRE). ACTH levels were measured hourly (4-8 a.m.) during a "conventional" hydrocortisone replacement therapy and during a therapy with dual-release hydrocortisone.

Results: Salient differences between the morning ACTH profiles under the "conventional" hydrocortisone replacement regimen with 10-5-5 mg/die compared to the single-dose regimen with $20 \mathrm{mg}$ dual-release hydrocortisone were not discernible.

Conclusion: DIRE could be an underestimated problem in immunotherapy and could put the patients at hazard. Especially in case of an endocrinological DIRE concerning the adrenocorticotrophic axis, life-threatening situations can arise for the patients. As for the special situation with M. Addison and MM, where hormonal feedback mechanisms may cause further problems beyond the normal hormonal replacement therapy, we observed no salient differences in the early morning ACTH profiles under different hydrocortisone replacement regimens.
\end{abstract}

KEY WORDS: immune checkpoint inhibitors, malignant melanoma, primary adrenal failure, MSH, ACTH, dual-release hydrocortisone, delayed immune-related events

Wiad Lek. 2020;73(2):396-400

\section{INTRODUCTION}

The therapy with immune checkpoint inhibitors (ICI) has revolutionized the treatment of metastatic malignant melanoma (MM) [1] and is also used in other malignancies such as advanced renal cell carcinoma, metastatic nonsmall cell lung cancer, squamous cell carcinoma of the head and neck (SCCHN), and tumors of the urinary tracts as well as progressive classical Hodgkin's lymphoma [2]. The growing use of these therapies sensitized physicians for a growing rate of adverse effects. These include rash, diarrhea, hepatitis, myocarditis and pneumonitis [3], but the immune-mediated adverse effects also include endocrinopathies such as hypophysitis, adrenalitis, thyroiditis, hypoparathyroidism and diabetes mellitus [4].

A decade ago, hyponatremia in MM patients - if not induced by drugs - could usually be recognized as a consequence of the syndrome of inappropriate antidiuretic hormone secretion (SIADH) [5]. Nowadays, hyponatremia as a typical electrolyte disturbance in hypocortisolism needs to be in the center of attention of the treating physicians immediately. Hypocortisolism, irrespective of its primary (adrenalitis, Addison's disease) or secondary (hypophysitis) nature, if not recognized early enough, can be a fatal event. This recently prompted the clinical committee of the Society for Endocrinology to publish a guideline for the acute management of the endocrine complications of checkpoint inhibitor therapy [6].

As for the primary or secondary nature of hypocortisolism, hypophysitis is more frequent than adrenalitis [7]. Interestingly, hypophysitis not related to ICI is an extremely rare event, whereas adrenalitis not related to ICI is rare, but much more frequent in comparison to hypophysitis.

A clarification of the etiology of hypocortisolism after or during ICI therapy is of importance, since patients with adrenalitis may not only require glucocorticoid replacement therapy, but also a replacement of mineralocorticoids (fludrocortisone), or replacement of dehydroepiandrosterone (DHEA) in some cases. Furthermore, the author recently expressed his concern [8] that the elevated levels of adrenocorticotrophic hormone (ACTH) and melanocyte-stimulating hormone (MSH) typical for primary adrenal failure (also sometimes under adequate substitution regimens and not only as a reason of incompliance) [9] might put MM patients at risk for an unfavorable course of this disease. This is because not only melanocytes, but also melanoma cells can express receptors for ACTH and 
the different subtypes of MSH, which can also have proliferative properties; ACTH itself undergoes proteolytic cleavage to a-MSH. The hypothesis was recently outlined in detail [8].

Adrenalitis during or after ICI therapy is still rare [7]. That said, and given the a.m. concerns, we took the opportunity to study the course of ACTH levels in the early morning hours under a "classic" hydrocortisone replacement regimen with 3 daily doses and under a single-dose dual-release hydrocortisone replacement therapy, since this is the timespan with the highest ACTH levels [11]. We were interested to see if the patient might have a substantial decrease in ACTH levels under the latter regimen, which could possibly be beneficial with the above-mentioned concerns in mind [8].

\section{THE AIM}

One aim of the report is to sensitize the reader for side effects of immunotherapy not only during, but also after immunotherapy has been stopped (DIRE). The report also addresses the possible risk of elevated ACTH levels (caused by ICI induced adrenalitis) in MM patients and strategies to counteract this problem.

\section{MATERIAL AND METHODS}

\section{CASE}

A 62-year-old Caucasian female patient was diagnosed with malignant melanoma on the right thigh in June 2015 in Norway (nodular type Breslow, thickness $5 \mathrm{~mm}$, Clark Level 4 pTNM: pT3b). The extended histology revealed no BRAF mutation (BRAF is a human gene [proto oncogene] encoding the protein B-Raf, which is involved in directing cell growth) but a NRAS mutation in codon Q6 / 1 (The NRAS gene encodes the N-Ras protein that is involved primarily in the regulation of cell division).

For the first time in March 2016, a computed tomography $(\mathrm{CT})$ of the thorax showed several pulmonary round lesions, the largest with a diameter of $8 \mathrm{~mm}$ in segment 5 , compatible with a metastatic disease. Another $3 \mathrm{~mm}$ focal defect could be detected in segment 2 .

The patient had been treated with a programmed cell death protein 1 (PT1) inhibitor (Pembrolizumab) since July 2016 - December 16. From the beginning of this therapy, she developed a large-scale vitiligo (Fig. 1). Later, the patient returned to Germany and the therapy was continued since October 2017. In June 2017, she developed hypothyroidism (no autoantibodies) and underwent substitution therapy with L-Thyroxin ever since. After pulmonary remission in terms of imaging, pembrolizumab was given in January 2018 for the last time.

Due to a cardiac decompensation, the patient was hospitalized from February 12th, 2019 until March 1st, 2019. In the cardiac echography, an apical kinetics disorder was detected, most likely from ischemic genesis. A CT of the thorax was also performed. It showed a very small round focus in segment 2 on the left and multiple focal pneumonic infiltrates in segments 2, 6 and 10. The differential diagnosis also included necrotic metastases.

Interestingly, in the initial laboratory, sodium was 118 $\mathrm{mmol} / \mathrm{l}$ and potassium $5.17 \mathrm{mmol} / \mathrm{l}(3.5-5.1 \mathrm{mmol} / \mathrm{l})$, but creatinine was also elevated with $201 \mu \mathrm{mol} / \mathrm{l}$ (44-80).

After cardiac recompensation and antibiotic therapy, the patient was discharged, but one week later, due to increasing physical exhaustion and dyspnea, the patient was admitted to our clinic. She was dizzy, unresponsive and sleepy.

In the initial laboratory report, sodium was $131 \mathrm{mmol} / \mathrm{l}$ and potassium $3.55 \mathrm{mmol} / \mathrm{l}$ (after previous diuretic therapy). Creatinine was still elevated with $175 \mu \mathrm{mol} / \mathrm{l}$ (44-80). C-reactive protein (CRP) was $256 \mathrm{mg} / \mathrm{l}(<5)$ and procalcitonin $1.90 \mu \mathrm{g} / \mathrm{dl}(<0.5)$. The patient was referred to the intensive care unit and intubated. A CT of the chest showed pulmonary congestion with pleural effusions on both sides as well as with encapsulated pleural effusion on the right lobe. Furthermore, it revealed round herds in the upper lobe on the right. The patient received an antibiotic therapy with piperacillin/tazobactam. With inflammatory parameters increasing further, the antibiosis was switched to meropenem and linezolid. Despite improvement of the inflammatory values and the pulmonary infiltrates, which allowed for an extubation, the marked reduction of vigilance persisted. Brain imaging, as well as neurological examination including lumbal puncture, revealed no pathological findings. Together with the persisting hyponatremia (and sometimes elevated potassium values) a measurement of serum cortisol and serum ACTH was performed. Serum cortisol was $<0.05 \mu \mathrm{g} / \mathrm{dl}(4.82-19.5)$, ACTH was $380.5 \mathrm{ng} / \mathrm{l}$ (4.8- 48.8).

The patient received $250 \mathrm{mg}$ hydrocortisone and was fully responsive and reinvigorated again after 3 days. To confirm the diagnosis of primary adrenal failure vs. secondary adrenal failure, the ACTH measurement was repeated while the patient was fasted before she received a reduced hydrocortisone dose of $100 \mathrm{mg}$ (ACTH 349.0 $\mathrm{ng} / \mathrm{l}$ ) intravenously and, when she was under a stable substitution regimen, with hydrocortisone $15-10-5 \mathrm{mg} / \mathrm{die}$ (ACTH $291.2 \mathrm{ng} / \mathrm{l}$ ). Interestingly, in the initially computed tomography of chest and abdomen, the adrenal glands were described as inconspicuous.

Hypopituitarism of other axes was excluded: we had also measured Prolactin (12.7 $\mu \mathrm{g} / \mathrm{l}$ (reference range 4.8-23.3), Insulin-like growth factor 1 (IGF-1) $(210.8 \mu \mathrm{g} / \mathrm{l}$ (reference range 41-168)), Luteinizing hormone (LH) (30.1 IU/L (reference range 7.7-58.5)) and Follicle stimulating hormone (FSH) (64.4 (reference range 18-153)).

The patient could be discharged in April 2019, the hydrocortisone was gradually reduced to $10-5-5 \mathrm{mg}$ and the patient reported good physical and mental performance. Her BMI was $22.1 \mathrm{~kg} / \mathrm{m}^{2}$. The blood pressure was normal, as were sodium and potassium. Fludrocortisone was not required. Under this "conventional" hydrocortisone replacement regimen, she took $10 \mathrm{mg} \mathrm{HC}$ at 8 a.m., $5 \mathrm{mg}$ at 12 a.m. and $5 \mathrm{mg}$ at 6:30 p.m. at home and also during our examination. 


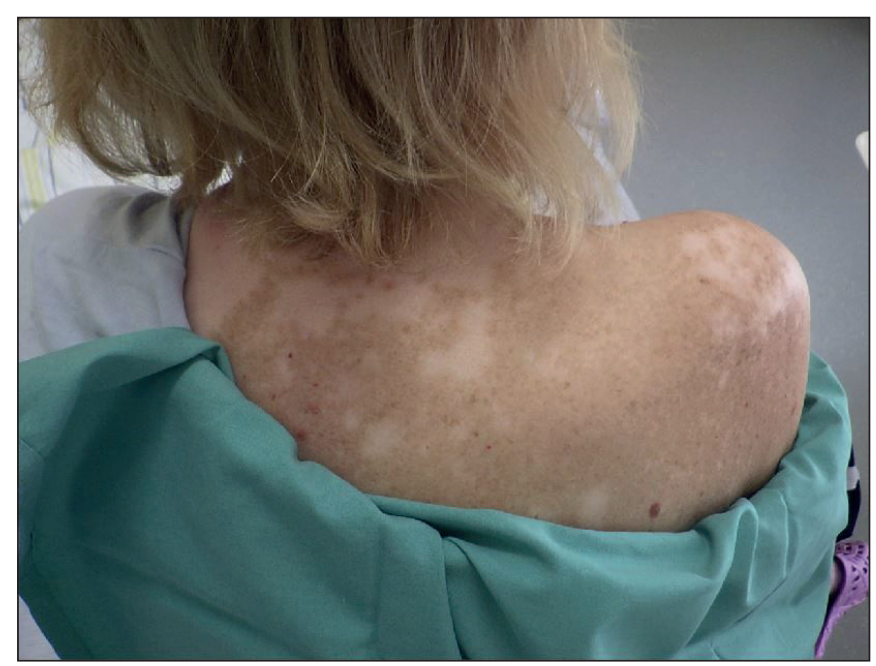

Fig. 1. Picture of the vitiligo on the back of the patient

\section{METHODS}

The blood sampling for ACTH was done in July 23th, 2019 under the conventional hydrocortisone replacement regimen at 4,5,6,7, and 8 a.m. To keep the blood sampling process as stress-free as possible, the sampling was done via the patient's venous port system. The measurements of ACTH were performed with a chemiluminescent immunoassay (CLIA) (LIAISON" ACTH assay; DiaSorin). The samples were measured in duplicate.

Then, the "conventional" hydrocortisone replacement regimen was changed to $20 \mathrm{mg}$ of dual-release hydrocortisone $\left(\right.$ Plenadren $\left.^{\mathrm{Tw}}\right)$ once per day, due to the concerns mentioned in the introduction. After a "wash in" phase of 7 days, under $20 \mathrm{mg}$ dual-release hydrocortisone, the blood sampling was done at the same times as under the conventional regimen (July 30th, 2019).

\section{RESULTS}

The profiles of ACTH are displayed in Figure 2. The low number of ACTH measurements in one single person precludes a statistical analysis. Salient differences between the morning ACTH profiles under the "conventional" hydrocortisone replacement regimen with $10-5-5 \mathrm{mg} / \mathrm{die}$ compared to the single-dose regimen with $20 \mathrm{mg}$ dual-release hydrocortisone were not discernible.

In the time after the switch, the patient had a stable weight, normal blood pressure and reported a slightly improved mental and physical performance. Thus, the therapy with the dual-release cortisone was maintained. In the most recent imaging, there was no hint for a recurrence of MM.

\section{DISCUSSION}

We describe the case of a patient in which several misleading findings delayed the establishment of a correct diagnosis of primary adrenal failure (large-scale vitiligo masked the typical hyperpigmentation of the skin, potassium levels normal after previous diuretic therapy, normal size of adrenal glands in abdominal imaging, "partial" empty sella syndrome known since 2017, the latter suggestive of a state after hypophysitis [12]. Primary adrenalitis after checkpoint inhibitor therapy is still a rare event. In July 2017, Tan and co-authors [7] screened 4 medical databases for adverse endocrine side events using checkpoint inhibitors. At this time, 451 patient cases were registered, with the undesirable side effects on the pituitary gland (222 cases) being the most prevalent. In most cases, this damage affected the adrenocorticotrophic axis, followed by secondary hypothyroidism and secondary sex hormone deficiency. Usually, several axes are affected. The primary adrenal insufficiency was reported in only 6 cases.

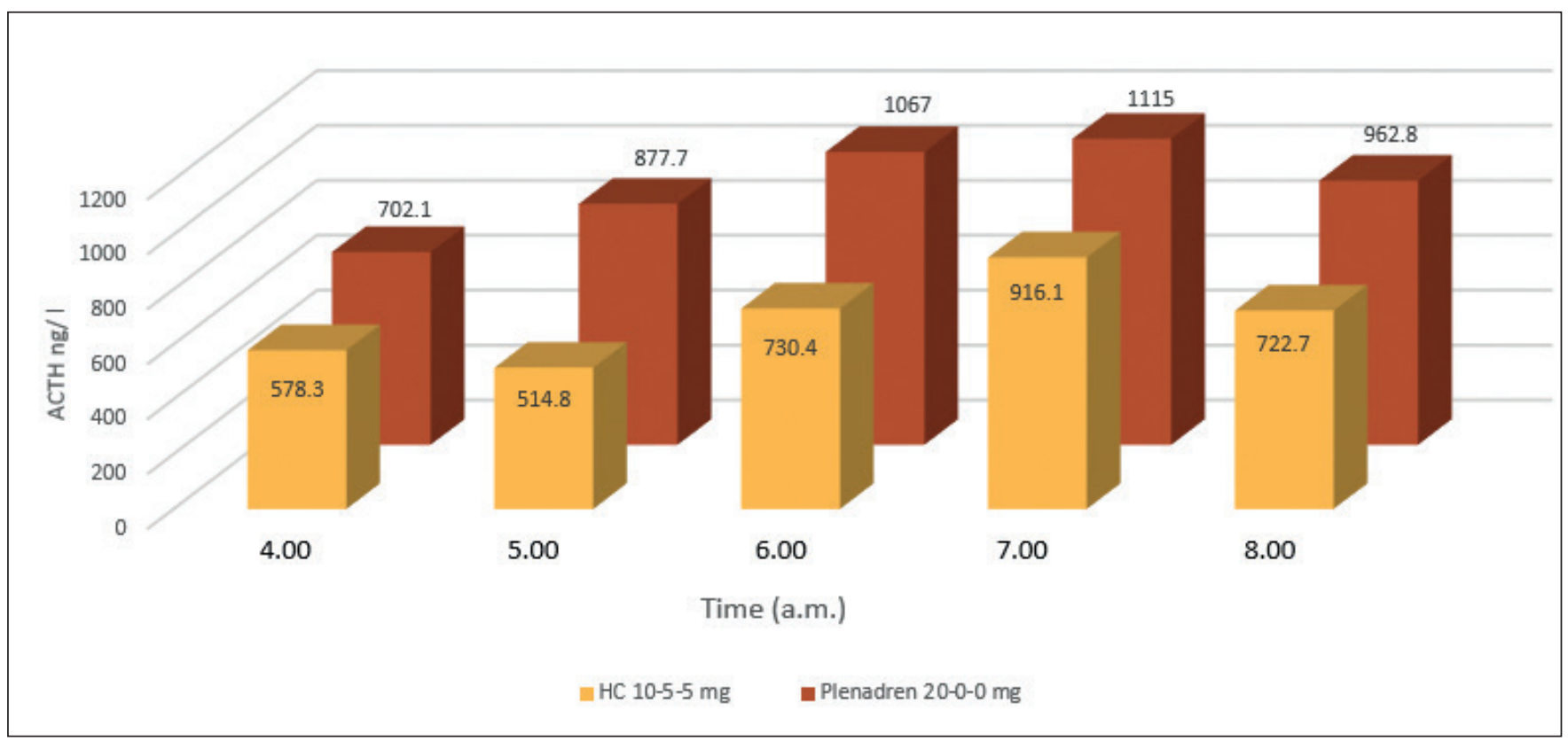

Fig. 2. Early-morning ACTH profiles of the patient.

ACTH levels (given in ng/l) during a "conventional" hydrocortisone replacement therapy with 10-5-5 mg (light brown), and ACTH levels during a therapy with dual-release hydrocortisone (20 mg once daily) (dark brown). The measurement times are displayed on the ordinate axis. 
A further pitfall in establishing the correct diagnosis in this case was that the delay between the last injection of pembrolizumab and the initial hospitalization was about one year. Recently, Couey et al. [13], after a pubmed research (January 2008 - December 2018), reported similar "delayed" effects of ICI in 23 published cases within a range of 1-18 months and with a median duration of 6 months. The authors named these adverse events after discontinuation of ICI "delayed immune-related events (DIRE)" and proposed a cutoff of 90 days post immunotherapy. In the 6 reported cases with adrenal failure, all of these were of a secondary nature. We could not identify reports from 2019 about delayed primary adrenal failure as a DIRE. That said, to our knowledge, we report the first clearly diagnosed case of delayed-onset primary adrenal failure here. So far, it is impossible to prove direct causality between immunotherapy and delayed autoimmunity. As for our patient, she reported no family history of autoimmune disease. Together with the rapid onset of vitiligo immediately after onset of the pembrolizumab therapy, this favors the assumption of a causality.

The "natural" course of CPI-induced adrenalitis in terms of imaging seems to be an initial enlargement of the adrenal glands, followed by a progressive loss of endocrine function and volume towards the initial size [14]. Interestingly, the authors in this paper reported, that hypophysitis and adrenalitis may coexist.

The measurement of ACTH and its levels has some pitfalls in terms of preanalytics [15] the time intervals of the measurements and the measurement itself. In eight healthy men, Veldhuis et al. [16] performed blood samplings of ACTH in 10-minute intervals for one day. The authors reported a half-life of endogenous ACTH of $15 \pm 1.2 \mathrm{~min}$. Furthermore, after applying a novel mathematical deconvolution model, this revealed $40 \pm 1.5$ significant ACTH secretory bursts $/ 24 \mathrm{~h}$. The mean interburst interval was reported to be $39 \pm 2.3 \mathrm{~min}$. The bursts had a half-duration (duration at half-maximal amplitude) of $19 \pm 2 \mathrm{~min}$. The authors defined half-duration as the duration of a half-maximal amplitude.

As for our measurements, there was no outlier suggestive of having collected ACTH during such a pulse. With regard to the reference values for ACTH given by the manufacturer of the assay, the measurements of ACTH in the early morning hours in our case impressively show the degree of elevation of these levels, irrespective of the patient being under a "conventional" hydrocortisone replacement regimen or under a single dose of dual-release hydrocortisone.

According to an Endocrine Society clinical practice guideline on "Diagnosis and Treatment of Primary Adrenal Insufficiency" [17], the authors suggest using hydrocortisone (15-25 mg) or cortisone acetate (20-35 mg) in two or three divided oral doses per day. Given the BMI of the patient (together with her wellbeing, the stable weight and blood pressure as well as the normalized sodium and potassium levels), there was no reason to assume an undersubstitution as a reason for the high ACTH levels.

There is also data about daytime ACTH measurements under both regimens: Recently, M. Addison patients who were treated with $20 \mathrm{mg}$ hydrocortisone (cumulative dose) thrice daily compared to a therapy with $20 \mathrm{mg}$ dual-release cortisone were assessed for variations of anthropometric, metabolic, and hormonal parameters. As for ACTH, it was reported that the decrease of ACTH levels was more marked and with a lower area under the curve in the latter group (18). That said, we did not see a necessity to retest these observations. However, these measurements of ACTH were done during daytime (baseline measurements beginning at 8-9 a.m., followed in 30 minute-intervals until $240 \mathrm{~min}$ after the last cortisone ingestion, which is usually 5 or 6 p.m. to avoid sleep disturbance under a conventional hydrocortisone regimen). Given a possible pathophysiological relevance of elevated ACTH and MSH levels and the lack of financial support, the focus of our interest was the "vulnerable" phase, the daytime when these levels are even physiologically the highest, which is in the early morning hours.

That said, with respect to the ACTH values, these values are lower during daytime. Our observation in a single case gives an indication that there may be no significant difference in the early morning hours irrespective of the substitution regime.

In order to confirm our observation, further studies seem sensible. These should be performed not only in larger patient groups, but with a closer monitoring of ACTH and also MSH levels. It would also be of interest to test them under a novel preparation of hydrocortisone $\left(\right.$ Chronocort $\left.^{\mathrm{mN}}\right)$ yet tested in phase III studies. This medication is taken at 11 p.m. and 7 a.m., not only with a more physiological profile of cortisol levels in mind, but also to reduce the early-morning rise of androgens in adrenogenital syndrome (AGS) [19]. However, it has to be pointed out, that those preparations are not designed with lower $\mathrm{ACTH}$ or MSH levels in mind.

\section{CONCLUSION}

A growing number of endocrine side effects during therapy with ICI is described in medical literature. This report highlights the further diagnostic hazard to identify these side effects not only during, but also after immunotherapy has been stopped. The maximal timespan in which such events are possible, is not yet known. As can be hypothesized in this very case, some hormonal feedback mechanisms may cause further problems beyond the normal hormonal replacement therapy. The report underlines the importance of a good cooperation between endocrinologists and oncologists.

\section{LIST OF ABBREVIATIONS}

ACTH: adrenocorticotropic hormone

AGS: adrenogenital syndrome

CLIA: chemiluminescent immunoassay

DIRE: delayed immune-related events

DHEA: dehydroepiandrosterone

ICI: immune checkpoint inhibitor

MM: malignant melanoma

MSH: melanocyte stimulating hormone 
SCCHN: squamous cell carcinoma of the head and neck SIADH: syndrome of inappropriate antidiuretic hormone secretion

\section{REFERENCES}

1. Hodi FS, O'Day SJ, McDermott DF, et al. Improved survival with ipilimumab in patients with metastatic melanoma. N Engl J Med. 2010;363:711-23.

2. Ansell SM, Lesokhin AM, Borrello I, et al. PPD-1 blockade with nivolumab in relapsed or refractory Hodgkin's lymphoma. N Engl J Med. 2015;372:311-9.

3. Brahmer JR, Tykodi SS, Chow LQ, et al. Safety and activity of antiPD-L1 antibody in patients with advanced cancer. N Engl J Med. 2012;366:2455-65

4. Ntali G, Kassi E, Alevizaki M. Endocrine sequelae of immune checkpoint inhibitors. Hormones (Athens). 2017;16:341-50.

5. Ueda $M$, Endo Y, Kaku Y, et al. The syndrome of inappropriate antidiuretic hormone secretion (SIADH) associated with metastatic malignant melanoma. Eur J Dermatol. 2012;22:411-2.

6. Higham CE, Olsson-Brown A, Carroll P, Society for Endocrinology Clinical Committee. SOCIETY FOR ENDOCRINOLOGY ENDOCRINE EMERGENCY GUIDANCE: Acute management of the endocrine complications of checkpoint inhibitor therapy. Endocr Connect. 2018;7:G1-G7.

7. Tan $M H$, lyengar $R$, Mizokami-Stout $K$, et al. Spectrum of immune checkpoint inhibitors-induced endocrinopathies in cancer patients: a scoping review of case reports. Clin Diabetes Endocrinol. 2019;5:1. doi: 10.1186/s40842-018-0073-4. eCollection 2019.

8. Harsch IA. Hypothesis: does adrenalitis caused by immune checkpointinhibitors put melanoma patients at an elevated risk for recurrence? J Immunother Cancer. 2019;7:166. doi: 10.1186/s40425-019-0651-8.

9. JeffcoateW. Assessment of corticosteroid replacement therapy in adults with adrenal insufficiency. Ann Clin Biochem. 1999;36:151-7.

10. Rosenkranz AA, Slastnikova TA, Durymanov MO, Sobolev AS. Malignant melanoma and melanocortin 1 receptor. Biochemistry (Mosc). 2013;78:1228-37. doi: 10.1134/\$0006297913110035.

11. Scott RS, Donald RA, Espiner EA. Plasma ACTH and cortisol profiles in Addisonian patients receiving conventional substitution therapy. Clin Endocrinol (Oxf). 1978;9:571-6.

12. Chang J, Tran J, Kamel D, Basu A. Nivolumab-induced hypophysitis leading to hypopituitarism and secondary empty sella syndrome in a patient with non-small cell lung cancer. BMJ Case Rep. 2019;12. pii: e228135. doi: 10.1136/bcr-2018-228135.

13. Couey MA, Bell RB, Patel AA, et al. Delayed immune-related events (DIRE) after discontinuation of immunotherapy: diagnostic hazard of autoimmunity at a distance. J Immunother Cancer. 2019;7:165. doi: 10.1186/s40425-019-0645-6.

14. Min L, Ibrahim N. I Iilimumab-induced autoimmune adrenalitis. Lancet Diabetes Endocrinol. 2013;1:e15. doi: 10.1016/S2213-8587(13)70031-7.

15. Lambert A, Frost J, Ratcliffe WA, Robertson WR. On the stability in vitro of bioactive human adrenocorticotrophin in blood and plasma. Clin Endocrinol (0xf). 1985;23:253-61.

16. Veldhuis JD, Iranmanesh A, Johnson ML, Lizarralde G. Amplitude, but not frequency, modulation of adrenocorticotropin secretory bursts gives rise to the nyctohemeral rhythm of the corticotropic axis in man. J Clin Endocrinol Metab. 1990;71:452-63.

17. Bornstein SR, Allolio B, ArltW, et al. Diagnosis and Treatment of Primary Adrenal Insufficiency: An Endocrine Society Clinical Practice Guideline. J Clin Endocrinol Metab. 2016;10:364-89. doi: 10.1210/jc.2015-1710.
18. Giordano R, Guaraldi F, Marinazzo E, etal.Improvement of anthropometric and metabolic parameters, and quality of life following treatment with dual-release hydrocortisone in patients with Addison's disease. Endocrine. 2016;51:360-8. doi: 10.1007/s12020-015-0681-z.

19. Jones CM, Mallappa A, Reisch N, et al. Modified-Release and Conventional Glucocorticoids and Diurnal Androgen Excretion in Congenital Adrenal Hyperplasia. J Clin Endocrinol Metab. 2017;102:1797-806. doi: 10.1210/j.2016-2855.

\section{ORCID and contributionship}

Igor Alexander Harsch - 0000-0003-2716-0066 A,B,D,E,F

Anastasiia Gritsaenko - B,F

Peter Christopher Konturek - ${ }^{A, E, F}$

\section{Declarations}

Ethics approval and consent to participate: The patient gave written permission to the early morning blood sampling. IAH is healthcare ethics consultant in the clinic and approved the blood sampling via the port system. Both therapyoptions (hydrocortisone and dual-release hydrocortisone) are approved for the therapy of Addison's disease.

\section{Consent for publication}

The patient gave written permission to publish her case and for the publication of the results of the early morning blood sampling.

\section{Availability of data and materials}

The datasets used and analyzed in this case are available from the corresponding author on reasonable request.

\section{Funding}

There was no funding

\section{Acknowledgements}

The author is grateful to Roman Harsch (BA Translation) for language editing and Andrea Ortloff (Endocrinologist assistant DGE) for her assistance with the early morning blood sampling and the graphic presentation.

\section{Conflict of interest}

None of the authors has a conflict of interests to declare.

\section{CORRESPONDING AUTHOR Igor Alexander Harsch, M.D.}

Thuringia Clinic Saalfeld "Georgius Agricola"

Rainweg 68, D-07318 Saalfeld/Saale

Tel.: +49[0]3671/541569;

TheFax.: +49[0]3671/541403

e-mail: iharsch@thueringen-kliniken.de

Received: 13.01 .2020

Accepted: 06.02.2020

A - Work concept and design, B - Data collection and analysis, C - Responsibility for statistical analysis,

$\mathbf{D}$-Writing the article, $\mathbf{E}-$ Critical review, $\mathbf{F}-$ Final approval of the article 